\title{
Cutting Balloon Angioplasty for the Prevention of Restenosis: Results of the Cutting Balloon Global Randomized Trial
}

\author{
Laura Mauri, MD, MSc, Raoul Bonan, MD, Bonnie H. Weiner, MD, \\ Victor Legrand, $M D$, PhD, Jean-Pierre Bassand, MD, Jeffrey J. Popma, MD, \\ Paulette Niemyski, RN, BSN, Ross Prpic, MD, Kalon K. L. Ho, MD, MSc, \\ Manish S. Chauhan, MD, Donald E. Cutlip, MD, Olivier F. Bertrand, MD, and \\ Richard E. Kuntz, MD, MSC
}

The cutting balloon (CB) is a specialized device designed to create discrete longitudinal incisions in the atherosclerotic target coronary segment during balloon inflation. Such controlled dilatation theoretically reduces the force needed to dilate an obstructive lesion compared with standard percutaneous transluminal coronary angioplasty (PTCA). We report a multicenter, randomized trial comparing the incidence of restenosis after CB angioplasty versus conventional balloon angioplasty in 1,238 patients. Six hundred seventeen patients were randomized to CB treatment, and 621 to PTCA. The mean reference vessel diameter was $2.86 \pm 0.49 \mathrm{~mm}$, mean lesion length $8.9 \pm 4.3 \mathrm{~mm}$, and prevalence of diabetes mellitus in patients was $13 \%$. The primary end point, the 6-month binary angiographic restenosis rate, was $31.4 \%$ for CB and $30.4 \%$ for PTCA ( $p=0.75$ ). Acute procedural success, defined as the attainment of $<50 \%$ diameter stenosis without in-hospital major adverse car-

$\mathbf{T}$ he search for a method to dilate an obstructive coronary lesion without invoking the proportional injury response observed in already approved devices ${ }^{1,2}$ led to the development of the cutting balloon (CB) (Boston Scientific Interventional Technologies, Natick, Massachusetts), an angioplasty balloon with 3 to 4 longitudinally bonded microtomes that is designed to score atherosclerotic plaque. It was hypothesized that the discrete longitudinal incisions created during balloon inflation might improve the success of angioplasty by reducing elastic recoil and minimizing intimal injury, thereby minimizing the neointimal proliferative response. ${ }^{3,4}$ Theoretically, this effect may

From the Brigham and Women's Hospital and Harvard Medical School, Boston, Massachusetts; Montreal Heart Institute, Montreal, Canada; University of Massachusetts, Worcester, Massachusetts; CHU Sart Tilman, Liège, Belgium; University Hospital Jean-Minjoz, Besançon, France; Harvard Clinical Research Institute, Boston, Massachusetts; Beth Israel Deaconess Medical Center, Boston, Massachusetts; Lahey Clinic Medical Center, Burlington, Massachusetts; and Quebec HeartLung Institute/Laval Hospital, Quebec, Quebec, Canada. This study was sponsored by Boston Scientific Interventional Technologies, Natick, Massachusetts. Manuscript received March 19, 2002; revised manuscript received and accepted June 27, 2002

Address for reprints: Raoul Bonan, MD, Montreal Heart Institute, 5000 Belanger Street, Montreal, Quebec HIT 1C8, Canada. E-mail: raoul.bonan@mmic.net. diac events, was $92.9 \%$ for CB and $94.7 \%$ for PTCA $/ p=$ 0.24). Freedom from target vessel revascularization was slightly higher in the CB arm $(88.5 \%$ vs $84.6 \%$, log-rank $p=0.04)$. Five coronary perforations occurred in the $C B$ arm only $(0.8 \%$ vs $0 \%, p=0.03)$. At 270 days, rates of myocardial infarction, death, and total major adverse cardiac events for CB and PTCA were $4.7 \%$ versus $2.4 \%$ $(p=0.03), 1.3 \%$ versus $0.3 \%(p=0.06)$, and $13.6 \%$ versus $15.1 \%(p=0.34)$, respectively. In summary, the proposed mechanism of controlled dilatation did not reduce the rate of angiographic restenosis for the $C B$ compared with conventional balloon angioplasty. CB angioplasty should be reserved for difficult lesions in which controlled dilatation is believed to provide a better acute result compared with balloon angioplasty alone. ( $) 2002$ by Excerpta Medica, Inc.

(Am J Cardiol 2002;90:1079-1083)

allow $\mathrm{CB}$ angioplasty to achieve and maintain a larger lumen diameter using lower balloon inflation pressures and durations than conventional percutaneous transluminal coronary angioplasty (PTCA). Moreover, by creating controlled incision planes, $\mathrm{CB}$ treatment may reduce rates of major dissection associated with angioplasty. ${ }^{5}$ Multiple case series from North America, Europe, and Japan - as well as small randomized studies-have suggested the safety and utility of CB angioplasty, ${ }^{4-10}$ and a reduction in restenosis in patients treated with CB compared with PTCA. ${ }^{5,11,12} \mathrm{We}$ present a large multicenter randomized clinical trial designed to determine the acute and late-term effects of $\mathrm{CB}$ angioplasty on obstructive coronary disease as compared with PTCA.

\section{METHODS}

Study design: The primary objective of this study was to evaluate rates of angiographic restenosis after $\mathrm{CB}$ angioplasty as compared with PTCA. The trial complied with the provisions of the Declaration of Helsinki, and all investigational sites received approval from their institutional review boards. Written informed consent was obtained from all patients.

Criteria for eligibility: Patients aged 25 to 75 years were eligible for the study if they had evidence of 
myocardial ischemia attributable to a de novo stenosis $<20 \mathrm{~mm}$ in length in a native coronary artery $\geq 2 \mathrm{~mm}$ in diameter. The principal clinical criteria for patient exclusion were a history of angioplasty of the target lesion, contraindication to emergency coronary artery bypass surgery, angina at rest or Q-wave myocardial infarction within the preceding 48 hours, and concomitant congestive heart failure. The angiographic criteria for patient exclusion were total occlusion, presence of thrombus, severe calcification, ulceration, vessel angulation of $>45^{\circ}$, and American College of Cardiology/American Heart Association class C lesions. ${ }^{13}$

Coronary intervention: Eligible patients were randomized to a strategy of $\mathrm{CB}$ or PTCA. For the CB strategy, a balloon-to-artery (diameter) ratio was limited to between 0.9 and 1.1, and a balloon length (10 or $15 \mathrm{~mm}$ ) to cover the proximal and distal margins of the lesion was chosen. Tandem inflations were performed for lesions 15 to $20 \mathrm{~mm}$ in length. At each site, a single $\mathrm{CB}$ inflation was performed with a final pressure of 4 to $8 \mathrm{~atm}$ for $\leq 90$ seconds. If a residual stenosis of $>40 \%$ was present after CB dilation, subsequent dilations were performed using a conventional angioplasty balloon. For the PTCA strategy, the final pressure and duration, and number of balloon inflations were left to the discretion of the operator. All patients received aspirin and intravenous heparin before angioplasty. Intracoronary nitroglycerin was administered before baseline and postintervention angiography.

Collection of data and analyses at the core laboratory: Case report forms were completed at each site, supervised by independent study monitors, and submitted to the data-coordinating center (Cardiovascular Data Analysis Center/Harvard Clinical Research Institute, Boston, Massachusetts). An independent clinical events committee that was unaware of each patient's treatment assignment adjudicated all major adverse cardiac events.

Angiograms obtained during the procedure and at the 6-month follow-up were submitted to the angiographic core laboratory (Washington Hospital Center Angiographic Core Laboratory, Washington, DC). Patients who developed recurrent symptoms of ischemia before the scheduled 6-month follow-up also underwent angiography. Quantitative angiographic analysis was performed using selected cine frames at baseline and at the end of the procedure, and at 6 months. A minimum of a 7Fr guiding catheter was used for accurate calibration. Reference and minimum lumen diameters were determined with an automated edgedetection algorithm (Cardiovascular Measurement System, Medis Medical Imaging Systems, Nuenen, The Netherlands). The acute gain, late loss, and lateloss index were defined as previously described.2,14

Creatine kinase (CK) and isoenzyme (CK-MB) determinations were performed at 8,16 , and 24 hours following completion of the procedure, and a 12-lead electrocardiogram was performed immediately after the procedure and before hospital discharge. Electrocardiograms were submitted to a core laboratory for analysis (Duke Clinical Research Institute, Durham,
North Carolina). Any patient with signs or symptoms of ischemia had additional CK and CK-MB determinations recorded.

Study end points: The primary end point for the present study was binary angiographic restenosis, defined as percent diameter stenosis $>50 \%$ at 6-month follow-up. The secondary end points included: acute procedural success, defined as a composite of attainment of a $<50 \%$ diameter stenosis in the absence of in-hospital major adverse cardiac events; acute device success, defined as the achievement of $<50 \%$ stenosis of the target lesion without crossover treatment or unplanned coronary stenting; target lesion revascularization, defined as clinically driven revascularization of the target lesion; target vessel revascularization, defined as clinically driven revascularization of any lesion in the target vessel; and myocardial infarction. Myocardial infarction was defined as $Q$ wave-the development of new, pathologic $\mathrm{Q}$ waves in $\geq 2$ contiguous leads with postprocedure CK-MB levels above normal, or as non-Q-wave-elevation of postprocedure $\mathrm{CK}$ levels to $>2$ times than normal, with CK-MB levels above normal in the absence of new, pathologic $\mathrm{Q}$ waves. The major adverse cardiac event rate, at 30 and at 270 days, was defined as the composite of death, Q-wave myocardial infarction, emergency bypass surgery, and target lesion revascularization. The loss index was defined as the slope of the linear regression model of late loss (dependent variable) and acute gain (independent variable). ${ }^{1}$

Statistical analysis: The present study was designed to have a power of $90 \%$ to reject the null hypothesis of no difference between the treatment groups with a 5\% level of significance in 2-tailed tests. On the basis of an expected 6-month restenosis rate of $40 \%$ for the conventional angioplasty strategy, and 30\% for the CB strategy (25\% treatment effect), and assuming $80 \%$ angiographic follow-up, a sample size of 1,244 patients was calculated.

All comparisons were performed in accordance with the intention-to-treat principle. Continuous variables were compared using Student's $t$ test if data were normally distributed and the Wilcoxon rank-sum test if they were not. Binary variables were compared using Fisher's exact test. Survival was estimated using the Kaplan-Meier method ${ }^{15}$ and compared using the log-rank test. A multivariable model of restenosis was constructed in which the dependent variable was binary angiographic restenosis at 6 months and the independent variables were selected baseline covariates. A 2-tailed $p$ value of $<0.05$ was considered to indicate significance. All analyses were performed using SAS software (version 6.12, SAS Institute, Cary, North Carolina). All data were analyzed according to the intention-to-treat principle.

\section{RESULTS}

Between April 1994 and November 1996, 1,245 patients were enrolled at 34 investigational sites in North America and Europe. A total of 1,238 patients were treated. Six hundred seventeen patients were randomized to CB treatment, and 621 to PTCA. Seven 
TABLE 1 Baseline Demographics and Clinical Characteristics

\begin{tabular}{|lcc|}
\hline Patient Characteristic & $\begin{array}{c}\mathrm{CB} \\
\text { (n = 617) }\end{array}$ & $\begin{array}{c}\text { PTCA } \\
\text { (n=621) }\end{array}$ \\
\hline Age (yrs) & $59 \pm 10$ & $58 \pm 11$ \\
Men & $72 \%$ & $77 \%$ \\
Smoking & $67 \%$ & $69 \%$ \\
Diabetes mellitus & $13 \%$ & $12 \%$ \\
Dyslipidemia & $45 \%$ & $47 \%$ \\
Hypertension & $34 \%$ & $37 \%$ \\
Prior myocardial infarction & $37 \%$ & $39 \%$ \\
Prior coronary artery bypass graft surgery & $3 \%$ & $3 \%$ \\
CCS class III or IV angina & $64 \%$ & $64 \%$ \\
\hline
\end{tabular}

Values are expressed as percentages (counts/sample size) or mean $\pm S D$. $\mathrm{CCS}=$ Canadian Cardiovascular Society.

TABLE 2 Baseline Angiographic Characteristics

\begin{tabular}{|lcc|}
\hline Lesion Characteristic & $\begin{array}{c}\text { CB } \\
(\mathrm{n}=689)\end{array}$ & $\begin{array}{c}\text { PTCA } \\
(\mathrm{n}=696)\end{array}$ \\
\hline Lesion length (mm) & $8.9 \pm 4.2$ & $8.9 \pm 4.4$ \\
Reference vessel diameter (mm) & $2.84 \pm 0.49$ & $2.87 \pm 0.49$ \\
Minimal lumen diameter (mm) & $0.96 \pm 0.33$ & $0.98 \pm 0.34$ \\
Diameter stenosis (\%) & $66 \pm 11$ & $66 \pm 11$ \\
Target vessel & $42 \%$ & $42 \%$ \\
$\quad$ Left anterior descending artery & $28 \%$ & $24 \%$ \\
Left circumflex artery & $31 \%$ & $34 \%$ \\
Right coronary artery & $14 \%$ & $15 \%$ \\
Calcification (moderate or severe) & $4 \%$ & $3 \%$ \\
Thrombus & $44 \%$ & $44 \%$ \\
Eccentric lesion & $6 \%$ & $5 \%$ \\
Angulation $>45^{\circ}$ & & \\
Lesion class* & $14 \%$ & $15 \%$ \\
A & $40 \%$ & $37 \%$ \\
B1 & $42 \%$ & $45 \%$ \\
B2 & $3 \%$ & $3 \%$ \\
C & & \\
\hline
\end{tabular}

Values are expressed as percentages (counts/sample size) or mean \pm SD. *American College of Cardiology/American Heart Association Lesion Class. ${ }^{13}$

patients were excluded after randomization but before treatment assignment because of failure to meet eligibility criteria.

Baseline characteristics: The baseline clinical and angiographic characteristics were similar in patients of the 2 treatment groups (Tables 1 and 2). Importantly, the 2 groups were well-matched with respect to variables known to increase the risk of restenosis-diabetes mellitus, Canadian Cardiovascular Society angina class III or IV, and location of lesion in the left anterior descending artery. ${ }^{16-18}$ The mean reference vessel diameter before the procedure was $2.84 \pm 0.49$ $\mathrm{mm}$ for $\mathrm{CB}$ and $2.87 \pm 0.49 \mathrm{~mm}$ for PTCA, whereas the mean lesion length was $8.9 \pm 4.2$ and $8.9 \pm 4.4$ $\mathrm{mm}$, respectively. Both groups had a high prevalence of current smokers. Most of the treated lesions were moderately complex (American College of Cardiology/American Heart Association grade B1 or B2). ${ }^{13}$

Acute procedural success: Acute procedural success was $92.9 \%$ for CB and $94.7 \%$ for PTCA $(p=0.24)$, and acute device success was also similar $(77.7 \%$ vs $77.8 \%, \mathrm{p}=1.0)$. The balloon-to-artery ratio employed was similar in the 2 study arms $(1.0 \pm 0.1$ vs $1.0 \pm$
$0.1)$. In addition, there were no significant differences in rates of dissection after the procedure (36\% vs $35 \%$, $\mathrm{p}=0.34)$ or "bail-out" stenting ( $13 \%$ vs $16 \%, \mathrm{p}=$ $0.13)$. However, CB left a smaller immediate minimal lumen diameter after the procedure $(2.05 \pm 0.52 \mathrm{vs}$ $2.13 \pm 0.53 \mathrm{~mm}, \mathrm{p}=0.01)$ and a greater residual percent diameter stenosis $(29 \pm 14 \%$ vs $27 \pm 13 \%$, p $=0.01)$ than PTCA (Table 3).

Angiographic follow-up: Repeat angiography was performed at 6 months in $551(82 \%)$ and $559(80 \%)$ of lesions treated with CB and PTCA, respectively. During the 6-month angiographic follow-up the primary end point of binary angiographic restenosis was not significantly different between the 2 groups (CB $31.4 \%$ vs PTCA $30.4 \%, p=0.75$; Table 3 ), and the follow-up minimal lumen diameter and percent diameter stenosis were also similar (Table 3).

A nonsignificant reduction in lower absolute late loss at 6 months in the CB arm $(0.43 \pm 0.61$ vs 0.50 $\pm 0.60, \mathrm{p}=0.06)$ failed to translate to lower angiographic restenosis rates because $\mathrm{CB}$ achieved a smaller acute gain than PTCA (1.09 \pm 0.54 vs $1.15 \pm$ $0.53, \mathrm{p}=0.04)$. In addition, the proportional response to injury, as measured by the late loss index, was not different between CB and PTCA arms $(0.49 \pm 0.04$ vs $0.44 \pm 0.05, \mathrm{p}=0.3$ ).

Clinical follow-up: At 270 days, clinical follow-up was available in 580 patients $(94 \%)$ assigned to $\mathrm{CB}$ and 572 patients $(92 \%)$ assigned to PTCA. The 30-day major adverse cardiac event rate was $3.7 \%$ for $\mathrm{CB}$ versus $2.7 \%$ for PTCA ( $\mathrm{p}=0.34)$, and the 270 -day major adverse cardiac event rate was $13.6 \%$ versus $15.1 \%$, respectively ( $p=0.47$; Table 4$)$. Freedom from target vessel revascularization, however, was higher in the group treated with CB $(88.5 \%)$ compared with PTCA $(84.6 \%$, log-rank $p=0.04$; Figure 1). In comparison to the PTCA arm, there was a higher incidence of myocardial infarction, largely non-Qwave, in the CB arm (4.7\% for CB vs $2.4 \%$ for PTCA, $\mathrm{p}=0.03)$ and higher mortality at 9 months $(1.3 \%$ vs $0.3 \%, p=0.06$; Table 4).

There were 6 cases of emergency bypass surgery in each arm of the study (Table 4). Five coronary perforations occurred in the $\mathrm{CB}$ arm, and none occurred in the conventional angioplasty arm $(0.8 \%, 0 \%, \mathrm{p}=$ 0.03 ). Of the 5 perforations, 2 were detected angiographically by the appearance of contrast extravasation into the periadventitial space without tamponade; however, 3 perforations were clinically important. One patient underwent successful pericardiocentesis for treatment of tamponade. Two other patients died following perforation, tamponade, and emergency coronary artery bypass graft surgery. Within the CB arm, the balloon-to-artery ratio was higher in the cases of perforation than in the cases without perforation (mean $1.4 \pm 0.4$, range 1.1 to 1.8 for perforations vs $1.0 \pm 0.1$ for nonperforations, $\mathrm{p}=0.05$ ).

Multivariable regression analysis: Despite adjustment for differences in baseline characteristics and minimal lumen diameter after the procedure in a multivariable model, treatment strategy (CB vs PTCA) 
TABLE 3 Quantitative Angiographic Analysis

\begin{tabular}{|lccc|}
\hline Lesion Characteristic & CB & PTCA & $p$ Value \\
\hline Immediatly after procedure & & & \\
Reference vessel diameter (mm) & $2.89 \pm 0.49$ & $2.93 \pm 0.48$ & 0.13 \\
Minimal lumen diameter (mm) & $2.05 \pm 0.52$ & $2.13 \pm 0.53$ & 0.01 \\
Residual (\%) diameter stenosis & $29 \pm 14$ & $27 \pm 13$ & 0.01 \\
Acute gain (mm) & $1.09 \pm 0.54$ & $1.15 \pm 0.53$ & 0.04 \\
At 6-mo follow-up & $2.79 \pm 0.46$ & $2.83 \pm 0.47$ & 0.15 \\
Reference vessel diameter (mm) & $1.63 \pm 0.62$ & $1.65 \pm 0.61$ & 0.44 \\
Minimal lumen diameter (mm) & $42 \pm 19$ & $42 \pm 19$ & 0.99 \\
Diameter stenosis (\%) & $31.4 \%$ & $30.4 \%$ & 0.75 \\
Binary restenosis rate (>50\% stenosis) & $0.43 \pm 0.61$ & $0.50 \pm 0.60$ & 0.06 \\
Late loss (mm) & \multicolumn{3}{l}{} \\
\hline Values are expressed as percentages (counts/sample size) or mean \pm SD. & \\
\hline
\end{tabular}

\begin{tabular}{|lccc|}
\hline \multicolumn{2}{|l}{ TABLE 4 Early and Late Adverse Events } & & \\
Event & CB & PTCA & \\
& $(\mathrm{n}=617)$ & $(\mathrm{n}=621)$ & $\mathrm{p}$ Value \\
\hline Early complications (within 30 days) & & & \\
Total major adverse cardiac events & $23(3.7 \%)$ & $17(2.7 \%)$ & 0.34 \\
Death & $4(0.6 \%)$ & 0 & 0.06 \\
Myocardial infarction & $26(4.2 \%)$ & $14(2.3 \%)$ & 0.05 \\
Q wave & $6(1.0 \%)$ & $6(1.0 \%)$ & 1 \\
Non-Q-wave & $20(3.2 \%)$ & $8(1.3 \%)$ & 0.02 \\
Emergency bypass surgery & $6(1.0 \%)$ & $6(1.0 \%)$ & 1 \\
Target lesion revascularization & $16(2.6 \%)$ & $16(2.6 \%)$ & 1 \\
Subacute closure & $8(1.3 \%)$ & $10(1.6 \%)$ & 0.81 \\
Vessel perforation & $5(0.8 \%)$ & 0 & 0.03 \\
Major vascular complications & $2(0.3 \%)$ & $1(0.2 \%)$ & 0.62 \\
Total complications (through 9 mo) & $84(13.6 \%)$ & $94(15.1 \%)$ & 0.47 \\
Total major adverse cardiac events & $8(1.3 \%)$ & $2(0.3 \%)$ & 0.06 \\
Death & $29(4.7 \%)$ & $15(2.4 \%)$ & 0.03 \\
Myocardial infarction & $9(1.5 \%)$ & $7(1.1 \%)$ & 0.63 \\
Q wave & $20(3.2 \%)$ & $8(1.3 \%)$ & 0.02 \\
Non-Q-wave & $6(1.0 \%)$ & $6(1.0 \%)$ & 1 \\
Emergency bypass surgery & $72(12 \%)$ & $92(15 \%)$ & 0.11 \\
Target lesion revascularization & & & \\
\hline
\end{tabular}

ongoing since the advent of balloon angioplasty in $1977 .{ }^{1}$ First proposed by Schwartz et al, ${ }^{19}$ the proportional injury model of balloon dilatation in porcine coronary arteries demonstrated that the healing neointimal response was proportional to the degree of underlying histologic injury created by the initial balloon dilatation. This model has been upheld in clinical trials of coronary devices, in which a consistent proportional relation has been observed between injury, measured as acute gain imparted by the device, and the healing injury response, measured as the 6-month late loss in lumen diameter. ${ }^{2,20}$

Despite a wide variety of mechanical approaches designed to enlarge coronary lumen size beyond balloon dilatation alone (including plaque atherectomy, ablative mechanical and laser atherectomy, and metal stenting), a common proportional vascular injury response appears to transcend the wide array of mechanical interventions. That is, the relation between 6-month late loss and initial acute gain in the target lesion lumen diameter for any given intervention, known as the late-loss index, ${ }^{1}$ has been consistently observed to be approximately $50 \%$.

We evaluated whether $\mathrm{CB}$ angioplasty could reduce rates of restenosis by its proposed mechanism of

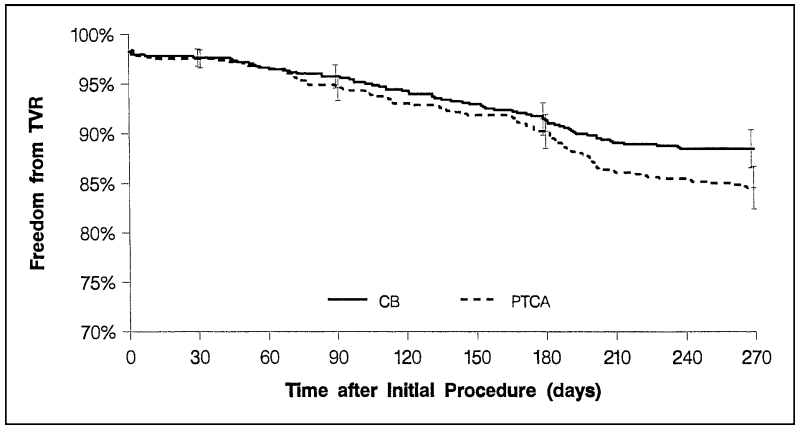

FIGURE 1. Kaplan-Meier estimated freedom from target vessel revascularization (TVR), demonstrating a higher survival for CB. ( $p=0.04)$.

was not a significant independent predictor of 6-month binary angiographic restenosis.

\section{DISCUSSION}

The search for a percutaneous coronary intervention that would relieve obstructive stenosis and minimize the inevitable tissue injury response that leads to restenosis in a sizeable minority of patients has been controlled injury causing minimal injury response. The results from this well-powered multicenter trial of 1,238 patients randomized to the $\mathrm{CB}$ versus PTCA demonstrated that the $\mathrm{CB}$ was associated with high rates of procedural success. However, there was no difference in the primary end point of binary angiographic restenosis between the 2 arms. This primary negative finding is well-supported by the trial design with $90 \%$ power to detect a $25 \%$ treatment effect.

The observation that the late loss index in the CB arm was no different from that of PTCA, even at the low pressures and durations used for $\mathrm{CB}$ inflation, suggests that the $\mathrm{CB}$ does not reduce the proportional response to injury. These results and the multivariable model are consistent with the conclusion that a larger minimum lumen diameter after the procedure is the most influential procedural predictor of restenosis, irrespective of the mechanism of plaque displacement. $^{2}$

It had been proposed that by creating controlled linear incisions, $\mathrm{CB}$ use could reduce the incidence of flow-limiting dissection after the procedure. In this study, however, rates of dissection were the same in both treatment groups. Perforation is a risk of all 
percutaneous coronary interventions. Five coronary perforations occurred after $\mathrm{CB}$ treatment $(0.8 \%)$ and none after conventional angioplasty $(\mathrm{p}=0.03)$. Given the small number of events, it is difficult to predict with certainty what procedural variables predispose to coronary perforation following CB treatment, but high balloon-to-artery ratios $(>1.1)$ were associated with this complication.

The role of $\mathrm{CB}$ may be for difficult lesions in which controlled dilatation is believed to provide a better acute result. Since the completion of this study, $\mathrm{CB}$ angioplasty has been proposed, with success in case series, for the treatment of resistant lesions, ${ }^{10}$ and also for the treatment of the in-stent restenotic lesions with or without vascular brachytherapy. ${ }^{21}$

In this multicenter, randomized clinical trial, $\mathrm{CB}$ angioplasty did not reduce rates of angiographic restenosis but did reduce the rate of target vessel revascularization. The CB demonstrated excellent acute procedural results; however, it is an example of another new coronary angioplasty device, that despite its novel mechanism of plaque displacement results, in the expected proportional response to injury previously seen with conventional angioplasty, atherectomy, and stenting. It is unlikely that different mechanical solutions will alter the arterial response to injury enough to prevent restenosis. To reduce the injury response, we will instead need to look forward to interventions that address the biology of restenosis, such as the promising drug-coated stents. ${ }^{22}$

1. Kuntz RE, Baim DS. Defining coronary restenosis. Newer clinical and angiographic paradigms. Circulation 1993;88:1310-1323.

2. Kuntz RE, Gibson CM, Nobuyoshi M, Baim DS. Generalized model of restenosis after conventional balloon angioplasty, stenting and directional atherectomy. J Am Coll Cardiol 1993;21:15-25.

3. Barath P, Fishbein MC, Vari S, Forrester JS. Cutting balloon: a novel approach to percutaneous angioplasty. Am J Cardiol 1991;68:1249-1252.

4. Popma JJ, Lansky AJ, Purkayastha DD, Hall LR, Bonan R. Angiographic, and clinical outcome after cutting balloon angioplasty. J Invasive Cardiol 1996; 8(suppl A):12A-19A

5. Ergene O, Seyithanoglu BY, Tastan A, Ergene U, Kozan O, Belgi A, Kaya D. Comparison of angiographic and clinical outcome after cutting balloon and conventional balloon angioplasty in vessels smaller than $3 \mathrm{~mm}$ in diameter: a randomized trial. J Invasive Cardiol 1998;10:70-75.

6. Unterberg C, Buchwald AB, Barath P, Schmidt T, Kreuzer H, Wiegand V.
Cutting balloon coronary angioplasty-initial clinical experience. Clin Cardiol $1993 ; 16: 660-664$

7. Hosokawa H. Cutting balloon angioplasty. A Japanese center's early experience. J Invasive Cardiol 1996;8(suppl A):20A-24A.

8. Kondo T, Kawaguchi K, Awaji Y, Mochizuki M. Immediate and chronic results of cutting balloon angioplasty: a matched comparison with conventional angioplasty. Clin Cardiol 1997;20:459-463.

9. Muramatsu T, Tsukahara R, Ho M, Ito S, Inoue T, Akimoto T, Hirano K. Efficacy of cutting balloon angioplasty for lesions at the ostium of the coronary arteries. J Invasive Cardiol 1999;11:201-206.

10. Bertrand OF, Bonan R, Bilodeau L, Tanguay JF, Tardif JC, Rodes J, Joyal M, Crepeau J, Cote G. Management of resistant coronary lesions by the cutting balloon catheter: initial experience. Cathet Cardiovasc Diagn 1997;41:179-184. 11. Molstad P, Myreng Y, Golf S, Sirnes PA, Kassis E, Abilgaard U, Andersen PE, Thuesen L. The Barath cutting balloon versus conventional angioplasty. A randomized study comparing acute success rate and frequency of late restenosis. Scand Cardiovasc J 1998;32:79-85.

12. Izumi M, Tsuchikane E, Funamoto M, Kobayashi T, Sumitsuji S, Otsuji S, Sakurai M, Awata N. Final results of the CAPAS trial. Am Heart J 2001;142: 782-789.

13. Smith SC Jr, Dove JT, Jacobs AK, Kennedy JW, Kereiakes D, Kern MJ, Kuntz RE, Popma JJ, Schaff HV, Williams DO, et al. ACC/AHA guidelines for percutaneous coronary intervention (revision of the 1993 PTCA guidelines)executive summary: a report of the American College of Cardiology/American Heart Association task force on practice guidelines (Committee to revise the 1993 guidelines for percutaneous transluminal coronary angioplasty) endorsed by the Society for Cardiac Angiography and Interventions. Circulation 2001;103:30193041

14. Kuntz RE, Hinohara T, Robertson GC, Safian RD, Simpson JB, Baim DS Influence of vessel selection on the observed restenosis rate after endoluminal stenting or directional atherectomy. Am J Cardiol 1992;70:1101-1108.

15. Kaplan EL, Meier P. Nonparametric estimation from incomplete observations. J Am Statist Assoc 1958;53:457-481.

16. Kuntz RE, Safian RD, Carrozza JP, Fishman RF, Mansour M, Baim DS. The importance of acute luminal diameter in determining restenosis after coronary atherectomy or stenting. Circulation 1992;86:1827-1835.

17. Kereiakes D, Linnemeier TJ, Baim DS, Kuntz R, O'Shaughnessy C, Hermiller J, Fink S, Lansky A, Nishimura N, Broderick TM, Popma J. Usefulness of stent length in predicting in-stent restenosis (the MULTI-LINK stent trials). Am J Cardiol 2000;86:336-341.

18. Bourassa MG, Lesperance J, Eastwood C, Schwartz L, Cote G, Kazim F, Hudon G. Clinical, physiologic, anatomic and procedural factors predictive of restenosis after percutaneous transluminal coronary angioplasty. J Am Coll Cardiol 1991;18:368-376.

19. Schwartz RS, Huber KC, Murphy JG, Edwards WD, Camrud AR, Vlietstra RE, Holmes DR. Restenosis and the proportional neointimal response to coronary artery injury: results in a porcine model. J Am Coll Cardiol 1992;19:267-274. 20. Baim DS, Cutlip DE, Sharma SK, Ho KK, Fortuna R, Schreiber TL, Feldman RL, Shani J, Senerchia C, Zhang Y, et al. Final results of the balloon vs optimal atherectomy trial (BOAT). Circulation 1998;97:322-331.

21. Eeckhout E, Roguelov H, De Benedetti E, Coucke P, SilberS, Albiero R, Schiele T, Bonan R, Urban P. Treatment of in-stent restenosis by combining cutting balloon angioplasty and vascular brachytherapy: a report from the RENO Registry. American College of Cardiology Scientific Session. Atlanta, GA, 2002. $J$ Am Coll Cardial 2002;5(suppl A):4A.

22. Morice MC, Serruys PW, Sousa JE, Fajadet J, Hayashi EB, Perin M, Colombo A, Schuler G, Barragan P, Guagliumi G, Molnar F, Falotico R. A randomized comparison of a sirolimus-eluting stent with a standard stent for coronary revascularization. $N$ Engl J Med 2002;346:1773-1780. 